\title{
Analysis of E- Plane Radiation Patterns of a Simulated Scalar Feed Horn Antenna
}

\author{
Dr. Stephen Rodrigues \\ Department of Instrumentation, Cochin University of Science \& Technology, Cochin-682 022, India.
}

\begin{abstract}
Simulated scalar feed horn antennas are found to be exhibiting identical radiation characteristics of metallic corrugated feed horn antennas of same dimensions. Hence, in this paper, the E-plane radiation characteristics of a simulated scalar feed horn antenna is analysed theoretically by extending the theory of metallic corrugated horn antennas. The theoretical E-plane radiation patterns at different frequencies in $X$ band are compared with that of experimentally obtained E-plane radiation patterns. Even though the nulls of of the radiation patterns are not fully realised by the theory, the main beam characteristics are well explained. Losses that may occur in the dielectric substrate of the E-plane walls are not considered in the theoretical explanation. This may be the reason for slight discrepancies observed in the experimental and theoretical 3-dB and $10 \mathrm{~dB}$ beam widths.
\end{abstract}

Key words: Antennas, Feed horns, Microwaves, Scalar feeds, Theoretical analysis

\section{Introduction}

The E-plane walls of the Simulated Scalar Feed (SSF) horn antenna are fabricated with dielectric substrate of appropriate thickness whose inner surface is periodically loaded with thin conducting strips which is called simulated corrugated surface $[1,2,3,4,5]$.It is well known that the E-plane aperture electric field distribution of a metallic corrugated antenna whose corrugation depth is judiciously selected for the balanced hybrid mode of operation is cosine in nature. The SSF horn antenna is also exhibiting a cosine nature for its Eplane aperture electric field distribution [3, 4]. Hence, the present theoretical analysis of the E-plane radiation characteristics of the SSF horn antenna is based on the theory of metallic corrugated horn antennas [6].

\section{I. Analysis of E- Field Distribution of E-Plane Aperture}

In order to calculate the radiation pattern, first the E- plane aperture electric field distribution of a square waveguide fabricated with simulated corrugated surface as its E-plane boundary walls is calculated. For this the theory of metallic corrugated horns [6] is used. Once the aperture electric field is obtained, the flaring of the horn in the two principal planes is accounted and the E-plane far field radiation patterns are calculated on the basis of vector diffraction formula.

The sketch of the square waveguide with strip grating structure on its E-plane boundary walls is shown in figure 1. The waveguide is directed along $\mathrm{z}$ - axis and $v$ and $\tau$ are the unit vectors which are respectively directed normally and tangentially to the boundary surface such that $\tau=I_{z} \times v$, where $I_{z}$ is the unit vector along $z-$ axis.

The surface impedance $Z_{\tau}$ and $Z_{z}$ are defined by the longitudinal field components $E_{\tau}$ and $H_{\tau}$ and are given by,

$$
\mathrm{Z}_{\tau}=\mathrm{E}_{\tau} / \mathrm{H}_{\mathrm{z}} \quad \text { and } \quad \mathrm{Z}_{\mathrm{z}}=\mathrm{E}_{\mathrm{z}} / \mathrm{H}_{\tau}
$$

Since at the boundary surface $E_{\tau}=0$, only the impedance $Z_{z}$ exists. However, as $Z_{z}$ is imaginary, a real parameter $\mathrm{Y}$ is introduced and it is defined as,

$$
\mathrm{J} Y=\mathrm{Z} / \mathrm{Z}_{\mathrm{Z}}
$$

Where $Z=\mu / \varepsilon \quad$ is the free space impedance. 

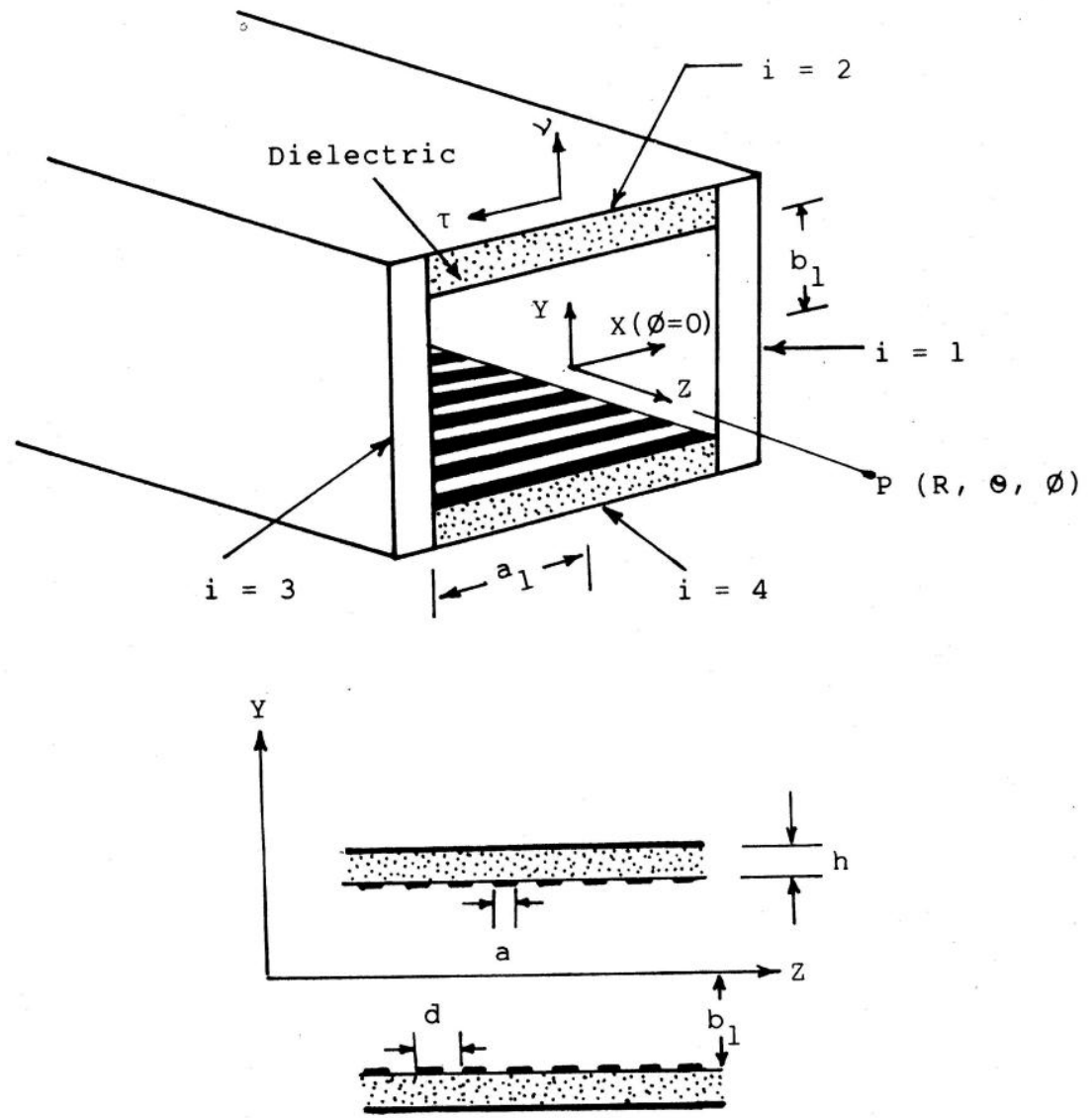

Fig.1. Sketch of the waveguide with strip grating structure on its dielectric E-plane walls

As per the indices given to the four walls of the waveguide in figure $1, Y_{n}$ denotes $\mathrm{Y}$ of the $\mathrm{n}$ Th wall. Since the opposite walls are identical in nature, $\mathrm{Y}_{1}=\mathrm{Y}_{3}$ and $\mathrm{Y}_{2}=\mathrm{Y}_{4}$. Now for a wave polarised in the $\mathrm{y}$ direction, $Y_{1}=\infty$. Then the transverse components of the electric field are given by,

$$
E_{x}=0 ; \quad E_{y}=\cos \alpha x \cdot \cos \gamma y e^{-j \beta z}
$$

Where $\alpha, \beta$ and $\gamma$ are the propagation constants.

Other field components can be determined using Maxwell's equations:

$$
\begin{aligned}
& E_{z}=j \omega \mu / \beta \cdot \cos \alpha x \cdot \cos \gamma y e^{-j \beta z} \\
& H_{x}=-1 / \beta \cdot\left(E \psi+\alpha^{2} / \omega\right) \cos \alpha x \cos \gamma y e^{-j \beta z} \\
& H_{y}=\alpha \gamma / \omega \beta \cdot \sin \alpha x \sin \gamma y e^{-j \beta z} \\
& H_{z}=j \alpha / \omega \cdot \sin \alpha x \cos \gamma y e^{-j \beta z}
\end{aligned}
$$

For the dominant $\mathrm{HE}_{11}$ mode,

$$
\alpha \mathrm{a}_{1}=\pi / 2
$$

As $E_{z}$ varies with $\mathrm{x}$ as $\cos \alpha \mathrm{x}$, we get

$$
\mathrm{Y}_{2}=-1 /(1-\mathrm{a} / \mathrm{d}) \cdot \gamma_{\mathrm{c}} / \mathrm{k} \tan \left(\gamma_{\mathrm{ch}}\right)
$$

Where $\gamma_{c}^{2}=k^{2}-\alpha^{2}$ 
$\mathrm{k}$ is the free space propagation constant. As stated earlier, for dominant $\mathrm{HE} 11$ mode, for large $\mathrm{ka}_{1} \mathrm{and} \mathrm{kb}_{1}$

$$
\alpha \mathrm{a}_{1}=\pi / 2 \quad \text { and } \quad \gamma \mathrm{b}_{1}=\pi / 2\left(1-\mathrm{Y}_{2} / \mathrm{kb}_{1}\right.
$$

In the case of a square waveguide, $a_{1}=b_{1}$.

Hence the aperture electric field of the waveguide under consideration is given by the equation,

$$
E_{y}=\cos \alpha x \cdot \cos \gamma y \quad e^{-j \beta z}
$$

\section{Analysis of Far- Field E- Plane Radiation Patterns}

Equation (7) is the E-plane aperture electric field distribution of the waveguide fabricated with strip loaded dielectric substrate as its E-plne boundary walls. In the case of a horn antenna, due to the flaring, a quadratic phase shift for the aperture field along the aperture is accounted by the factor $\mathrm{e}^{-\mathrm{j} 4 \pi \mathrm{y} 2 / \lambda \mathrm{L}}$. Incorporating this quadratic phase variation, the E- plane aperture electric field of the horn antenna is given by,

$$
E_{y}^{\prime}=\cos \alpha x \cdot \cos \gamma y \quad e^{-j \beta z} \quad e^{-j 4 \pi y 2 / \lambda L}
$$

In the above expression, $\mathrm{L}$ is the distance from the phase centre to the aperture of the horn. As a rectangular to square waveguide transition is used to feed the SSF horn antenna, the effective phase centre of the SSF horn antenna along with the transition is experimentally determined by plotting its phase pattern for different axes of rotation. The E-plane radiation pattern of the SSF horn antenna along with its phase pattern plotted for at an axis of rotation of $\mathrm{L}=18 \mathrm{~cm}$ at $10 \mathrm{GHz}$ is given in figure 2 .

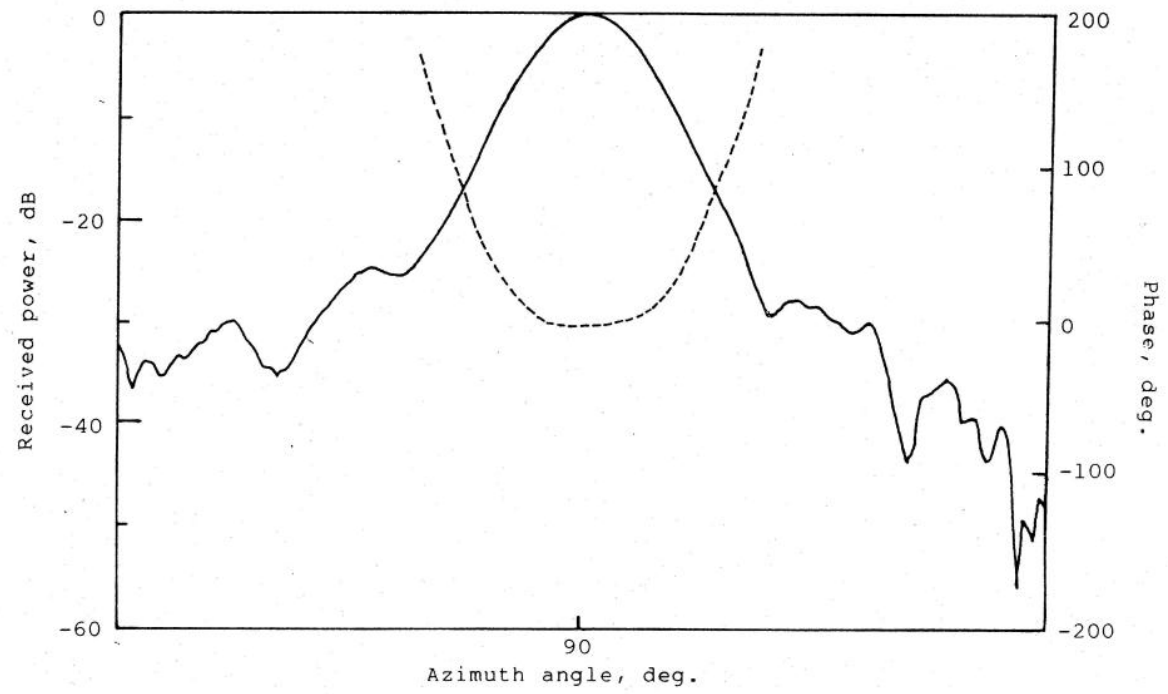

Fig. 2. The E- plane amplitude and phase patterns of SSF horn antenna plotted for at an axis of rotation of $\mathrm{L}=18 \mathrm{~cm}$ at $10 \mathrm{GHz}$.

Phase

The far-field radiation pattern in the E-plane $(\varphi=\pi / 2)$ of the simulated scalar feed horn antenna, based on the vector diffraction formula $[7,8,9]$ is given by,

$$
E_{\theta}=j k \exp (-J k R) / \pi R\left(1+k_{r} \cos \theta / k\right) \iint E_{y}^{\prime} \exp (j k y \sin \theta) d x d y
$$

Where $\mathrm{k}_{\mathrm{r}}=\left(\mathrm{k}^{2}-\pi^{2} / 4 \mathrm{a}_{1}^{2}\right)^{1 / 2}$ 


\section{Results}

The theoretically calculated E- plane radiation patterns compared with the experimental ones at different frequencies in X-band are presented in figures 3 and 4. The theoretically obtained 3-dB and 10- $\mathrm{dB}$ beamwidths of the SSF horn antenna compared with that of the experimental ones are given in table 1. From the table it is clear that the theoretical beamwidths are almost agreeing with the experimental values. In the theoretical calculations, the fields within the dielectric substrate are neglected. This may be the reason for the small discrepancies between the theoretical and experimental radiation patterns. Even though the theoretical calculations cannot predict the nulls of the radiation patterns exactly, the main beams are well predicted with a good accuracy.
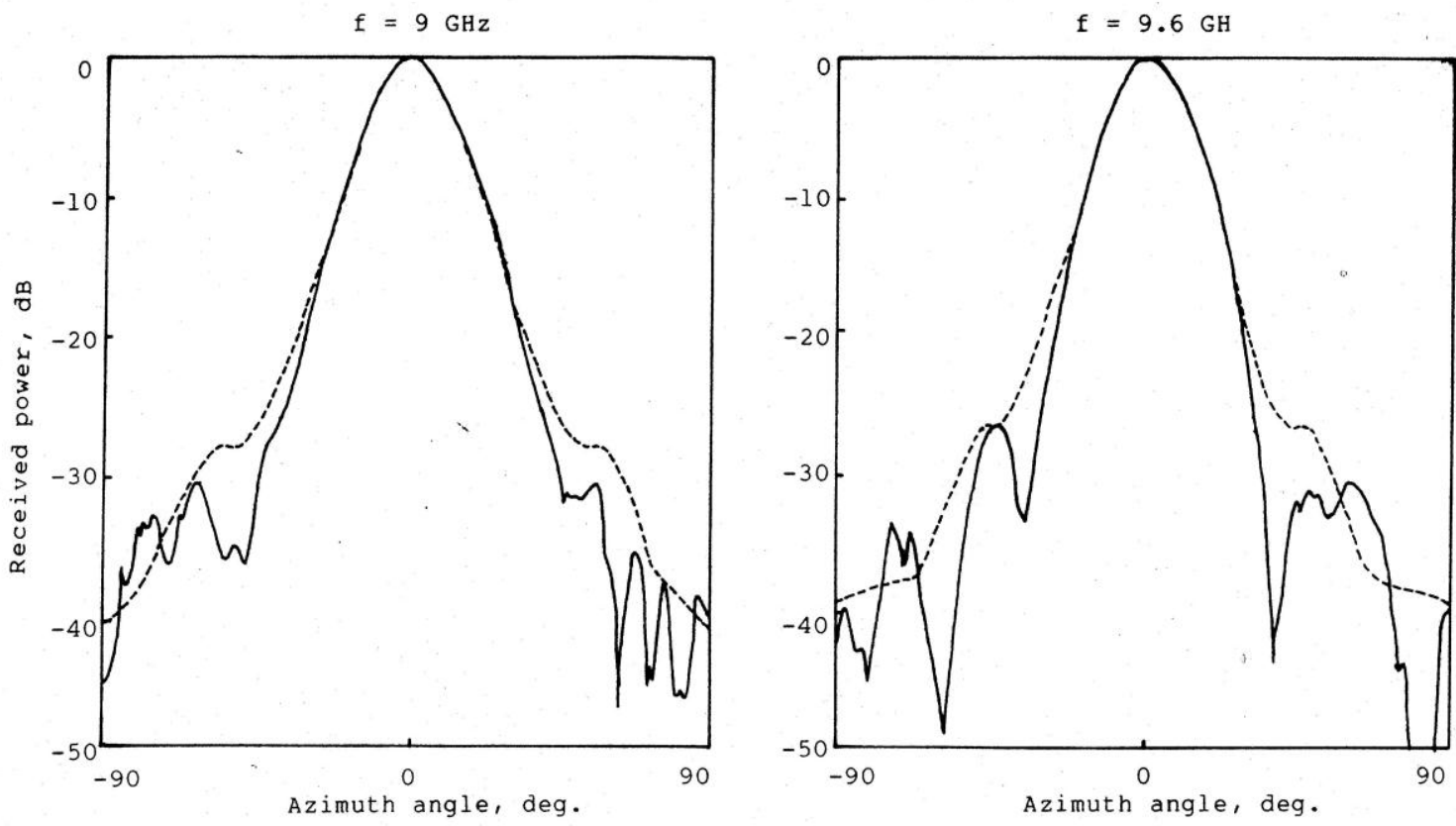

$\mathrm{f}=10 \mathrm{GHz}$

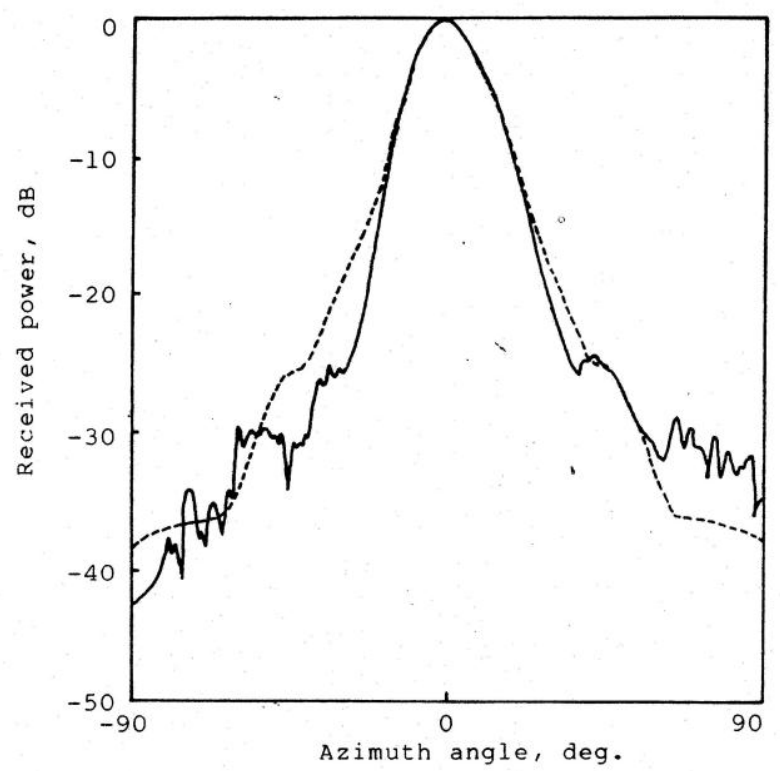

$f=11 \mathrm{GHz}$

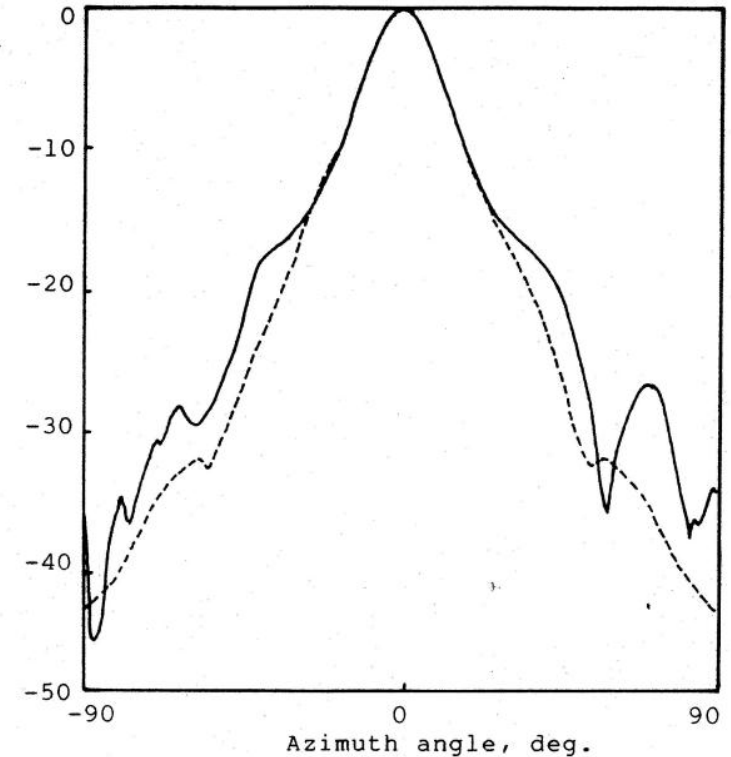

Fig.3. E-plane patterns of SSF horn antenna at different frequencies in X-band 
Table 1. Comparison of theoretical and experimental 3-dB and 10-dB beamwidths of the SSF horn antenna

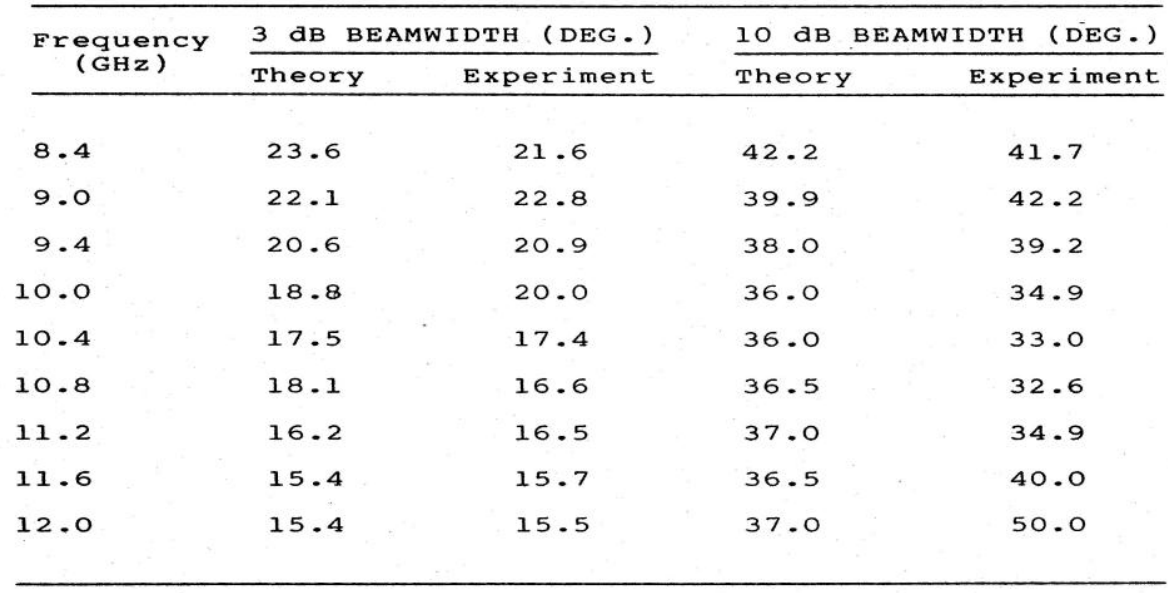

\section{References}

[1] E. Lier and T.S Petterson, “The Strip Loaded Hybrid Mode Feed Horn”, IEEE Trans. Antennas Propagat. Vol.AP-36, No. 9, pp. 1086- 1088, Sept.1987

[2] E. Lier and P.S Kildal, "Soft and Hard Horn Antennas", ibid, Vol. 36, No.8, pp. 1152-1157, Aug. 1988

[3] S. Rodrigues, P.Mohanan and K.G Nair, "Simulated Corrugated Feed Horn Antennas", Proc. IEEE AP-S Intl. Symp. USA, 1990, pp.984-987

[4] S. Rodrigues, P.Mohanan and K.G Nair, "A Strip Loaded Feed Horn Antenna", IEEE Microwave and Guided Wave Letters", Vol. 1,No.11, pp.318-319, Nov.1991

[5] S. Rodrigues, "Simulated Scalar Feed Horn Antenna with Exterior Tapered Throat Profile", IOSR Journal of Applied Physics", Vol.3, Issue 5, Mar-Apr., 2013, pp. 92-96

[6] C. Dragone, "A rectangular horn of four corrugated plates “, IEEE Trans. Antennas. Propagat. Vol. AP - 33, No. 2 , pp 160 - 164 , Feb. 1985 .

[7] M.S Narasimhan and V.V Rao, "Radiation from Wide Flare Corrugated E- Plane Sect oral Horn “, IEEE Trans. Antennas. Propagat. Vol. AP - 22, No. 2, pp 603 - 608, July 1974.

[8] S .Silver, "Microwave Antenna Theory and Design “, McGraw Hill Book Co., New York, 1949.

[9] R.E Collin and E.J Zucker, "Antenna Theory Part- I ", McGraw Hill Book Co., New York, 1969.

[10] S. Rodrigues, "A Small Square Aperture Simulated Corrugated Feed Horn Antenna with Beam Symmetry", IOSR Journal of Electronics And Communication Engineering “, Vol.8, Issue 3, Nov. - Dec. . . 2013, pp. 21 - 24

[11] S. Rodrigues, "Development of a New Reflecting Surface for Electromagnetic Waves", IOSR Journal of Applied Physics", Vol.1, Issue 6, Sep - Oct, 2012, pp. 55 - 58 